Original Research Paper

\title{
Bionic Technology and Deep Learning in Agricultural Engineering: Current Status and Future Prospects
}

\author{
${ }^{1,2}$ Chunlei Tu, ${ }^{3}$ Jinxia Li, ${ }^{1}$ Xingsong Wang, ${ }^{1,4} \mathrm{Cheng}$ Shen and ${ }^{1} \mathrm{Jie} \mathrm{Li}$ \\ ${ }^{1}$ School of Mechanical Engineering, Southeast University, Nanjing 211189, P. R. China \\ ${ }^{2}$ Special Equipment Safety Supervision Inspection Institute of Jiangsu Province, Nanjing 210036, China \\ ${ }^{3}$ Institute of Agricultural Remote Sensing and Information, \\ Heilongjiang Academy of Agricultural Sciences, Harbin 150086, China \\ ${ }^{4}$ Nanjing Institute of Agricultural Mechanization, Ministry of Agriculture and Rural Affairs, Nanjing 210014, China
}

\author{
Article history \\ Received: 23-02-2021 \\ Revised: 20-03-2021 \\ Accepted: 26-03-2021 \\ Corresponding Author: \\ Jie Li \\ School of Mechanical \\ Engineering, Southeast \\ University, Nanjing 211189, P. \\ R. China \\ E-mail: 1j2018576590@163.com
}

\begin{abstract}
As one of the most important production activity of mankind, agriculture plays an important role in social development. With the development of science and technology, agricultural technology has constantly been explored and researched. By learning and imitating the characteristics of creatures in nature, bionic technology has been applied to the improvement of agricultural machinery and farm implements. In recent years, as an extension of bionic technology, machine vision and deep learning have been widely used in agricultural production. The application of bionic technology and deep learning in agricultural engineering are reviewed in this study. In traditional agricultural engineering, many bionic farming tools were developed to reduce soil resistance and multiple bionic cutting cutters were designed to improve work efficiency and save energy. Machine vision and neural networks were widely used in crop classification, sorting, phenological period recognition and navigation. Deep learning methods can promote the intelligentization of agricultural engineering and has obvious advantages in crop classification, disease and pest identification, growth status evaluation and autonomous robots. Agricultural engineering that integrates bionic technology, machine vision and deep learning will develop toward more automation and intelligence.
\end{abstract}

Keywords: Counter-Regulatory Arms, RAS, Cardiovascular and Renal Function Bionic Technology, Deep Learning, Agricultural Engineering, Machine Vision

\section{Introduction}

Agriculture, as one driving force of human development, is the foundation for supporting social stability. Agricultural engineering has always been an important research field and various technologies have been continuously promoted and applied in agricultural engineering (Zhang et al., 2002). Bionic technology, with unique design thought, has a positive effect on the development of agricultural engineering. The birth of bionics opened a new era of human learning from nature (Luquan and Yunhong, 2016). "Bionics is the study of the structure, properties, principles, behaviors and interactions of biological systems, so as to provide new design ideas, working principles and system composition for engineering technology" was proposed by Academician (Yongxiang, 2004). So far, there is no unified and clear definition of bionics in the strict sense, but its basic connotation is clear: Bionics improves modern technical equipment and creates new technologies by studying and imitating various characteristics of the biological world (Dickinson, 1999), such as matter, energy, information, etc.

By imitating the characteristics and movements of various creature, bionics is an important form of human learning from nature. Bionic technology has been applied to optimized agricultural machineries and farm implements, which greatly improves the work efficiency of agricultural production (Jia et al., 2019; Li et al., 2017; Liu et al., 2014). Various bionic agricultural machineries have been designed and developed, such as tillage plows, cutting cutters and driving wheels.

Machine vision imitates human visual functions through computers and artificial neural networks imitate 
brain synaptic connections for information processing, both of which are new extensions of bionic technology. Machine vision and neural networks have been widely used in agricultural engineering, such as the classification and sorting of agricultural products, the estimation of the state of crops and the navigation of agricultural machinery. Machine vision has successfully replaced the recognition of human eyes, which can save labor and improve recognition accuracy (Patel et al., 2012; Xue et al., 2012). With the development of technology, more and more agricultural machineries are equipped with vision systems for intelligent monitoring, early warning, processing and other functions.

Deep learning (LeCun et al., 2015; Schmidhuber, 2015), as more complex neural networks in the field of machine learning, realizes more intelligent recognition and processing and promotes the development of artificial intelligence. In recent years, deep learning has been researched and promoted in the field of agriculture, which helps to improve the intelligence of traditional agriculture. In the process of agricultural production, deep learning performs well in crop classification, disease and pest diagnosis, growth status evaluation and autonomous robots. This paper systematically introduces the application status of bionic technology, machine vision, neural network and deep learning in agricultural engineering and considers and prospects the future development of bionic technology and deep learning.

\section{Bionic Application of Agricultural Machineries}

In the field of agricultural engineering, the interaction between soil and machinery is one of the research hotspots. Since the 1980 s, Academician Ren and his team conducted a systematic and in-depth study of earthworms and constructed the mechanical principles of bionic mollusk movement (Luquan and Yunhong, 2016). Based on some biological phenomena, such as earthworms build a lubricating interface film on their body surface through secreted mucus and surface electroosmosis to reduce soil adhesion and the nonsmooth surface structures of some soil animals have good performance in desorption and drag reduction, bionic desorption theory was gradually summed up.

\section{Soil Cultivation}

In the process of agricultural machinery touching the soil, the interaction between the soil and the touching parts brings greater resistance and power consumption and bionics is widely used in the resistance and consumption reduction technology of the touching parts. Generally, non-smooth surfaces on soil-touching parts of many soil animals have become bionic research objects, such as convex hulls, pits (Xu et al., 2009), ribs, scales (Li et al., 1998), which have the characteristics of desorption and drag reduction. Reducing abrasion and tillage resistance is the main goal of the improvement research of agricultural farming machinery. Zhang (2014) used reverse engineering technology to extract the ribbed geometric structure information on the outer surfaces of shell organisms and fit mathematical models to optimize the structures of subsoiling shovels. The test results indicate that the bionic ribbed subsoiling shovel has lower tillage resistance and better wear resistance. Xue (2017) designed a bionic coupling disc by analyzing the drag reduction characteristics of the dung beetle head and the pangolin back-surface, which has a better drag reduction performance and energy-saving effect.

As shown in Fig. 1, inspired by high excavation efficiency of soil burrow animals, bionic researches on the claws of these animals have made abundant achievements. Wang et al. (2018) designed a bionic weeding shovel based on the geometric structure of the zokor paw, which has a significant drag reduction effect when weeding among seedlings. Based on the profile curve characteristics of the mole's forefoot paw and toe, (Ji et al., 2012) designed a bionic rotary tillage cutter, which meets industry standards and consumes less power (Jia et al., 2009; Tong et al., 2015). The characteristics of soil animal surfaces, such lubrication, electroosmosis, have multiple coupling effects on reducing the surface viscosity and soil resistance. Zhang et al. (2019b) designed a bionic injection device to improve the performances of desorption and drag reduction during operation, using coupling of earthworm movement, mucus secretion and body surface structure as the bionic object, as shown in Fig. 2.

\section{Agricultural Robots}

The latest agricultural robots have integrated information technology, sensor technology and artificial intelligence technology on traditional agricultural machinery. Because field operations are different from production of industrial assembly line, the walking devices of agricultural robots are special.

Combining the buffalo striding strategy and the characteristics of wheel movement, Chen et al. (2003) proposed a bionic walking-wheel method, replacing the wheel rolling with walking on soft ground and it changed the mechanical structure system of coexisting load bearing and driving in traditional production. The MIT Biomimetic Robotics Lab took cheetahs as bionic objects and developed a cheetah-like robot with smoother movement (Ananthanarayanan et al., 2012; Seok et al., 2013). As shown in Fig. 3, Yanhu et al. (2018) designed an adaptive low-vibration walking wheel that mimics the foot motion posture of ostriches and the energy-storage and shock-absorption function of its metatarsophalangeal joints. 


\section{Agricultural Cutters}

Bionics provides new ideas and methods for the research, design and optimization of crop cutters. Tian et al. (2017) designed a blade that mimics the mouthparts of longhorn beetles, as shown in Fig. 4. In cutting tests of single-stalk hemps, its average maximum cutting force and cutting power consumption were reduced by 7.4 and $8.0 \%$, respectively. Wei et al. (2018) designed a bionic harvest cutting table of flax by capturing the upper-jaw contour of the Korean beetle and its cutting effect is obviously superior to that of the traditional standard cutter, reducing the cutting blank area by $50 \%$ and the recut area by $80 \%$. By imitating the chewing teeth of locusts, Honglei et al. (2018) designed a bionic saw blade for corn stalk cutting (Jia et al., 2013), a bionic disc cutter
(Wang et al., 2014), a dynamic bionic stubble cutting device (Honglei et al., 2018). The test results indicate that the power consumption of the bionic saw blade and bionic disc cutter are reduced by 12.85 and $18.49 \%$ respectively and the torque output of the bionic stubble cutting device is reduced by $19.5 \sim 21.8 \%$. Berling and Rechberger (2007) designed a plastic cutter based on the self-sharpening characteristic of beaver incisors and its wear rate was significantly reduced.

The above research demonstrates that bionic cutters can increase the service life, efficiency and reduce power consumption of the blades through the bionic design of animal shape and function. The application of bionic technology can better improve the shortcomings of traditional blades by learning from the advantages of biological evolution.

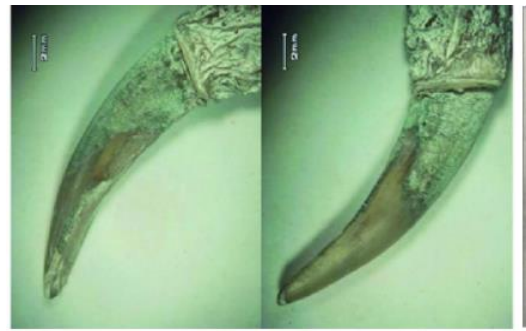

(a)

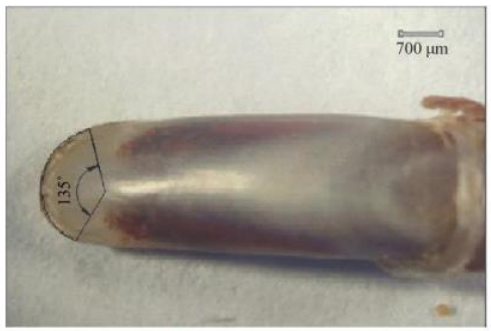

(b)

Fig. 1: Bionic objects. (a) the zokor paw (b) the toe of mole rat

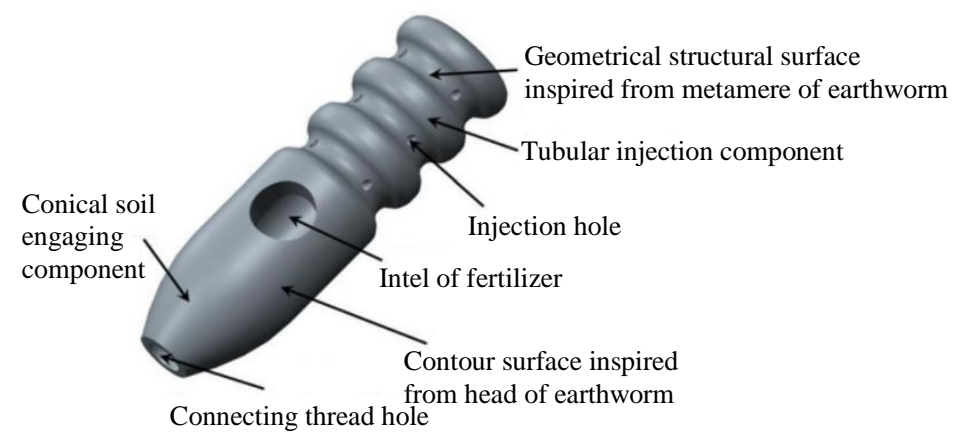

Fig. 2: Bionic injection device
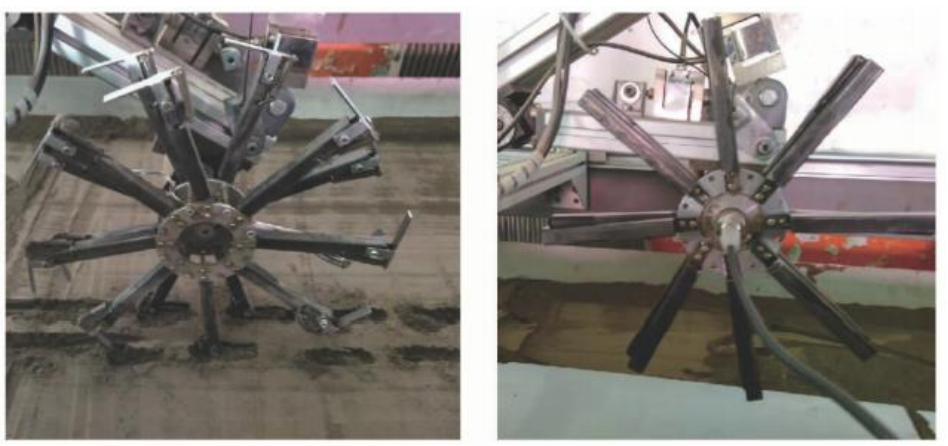

Fig. 3: Bionic walk wheel 


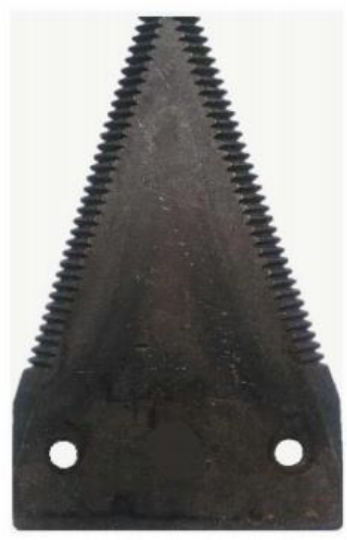

a

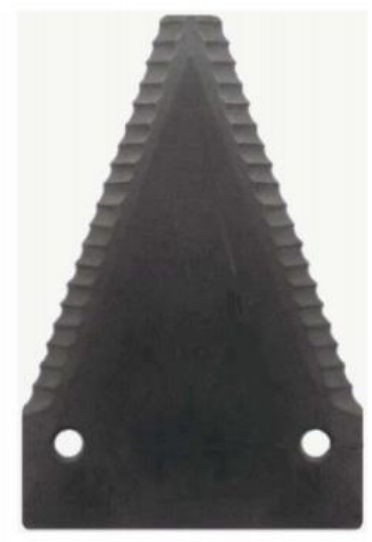

b
Fig. 4: (a) Ordinary blade; (b) Bionic blade

\section{Machine Vision and Neural Networks in Agricultural Engineering}

After the early bionic research on animal characteristics, bionic technology in the field of agricultural engineering has developed from ground mechanical drag reduction, bionic wear-resistant materials and bionic design to the direction of intelligent agriculture and advanced manufacturing. As the "intelligent eye" of artificial intelligence, machine vision plays an important role in the classification of agricultural products, the monitoring of weeds and pests and intelligent agricultural machinery, which has attracted the attention of many researchers. In recent years, artificial intelligence is the frontier development of bionics and many applied researches of artificial intelligence have been carried out in the field of agricultural engineering.

\section{Recognition and Classification}

In order to overcome the influence of color saturation, illumination and reflection on visual classification, Kang and Sams (1999) used artificial neural networks to train the color and shape of oranges to achieve recognition and classification. The test results indicate that this method has higher requirements for environmental conditions and the visual environment directly affects the recognition results.

Rocha et al. (2008; 2010) constructed different classifiers by extracting the color, texture and shape features of different fruits. They found the optimal combination to optimize the recognition rate and Fig. 5 shows average accuracy per class. They found that the combination of Color Coherence Vectors (CCVs), Border/Interior (BIC) and Sum and difference histograms (Unser) provides better average accuracies when considering intra-class variability. Seng and Mirisaee (2009) proposed a new fruit recognition system, which combines three features analysis methods: Color-based, shape-based and size-based in order to increase accuracy of recognition. The recognition results of the system are accurate up to $90 \%$.

In this regard, (Faria et al., 2012) used different classifiers to integrate different features to achieve "oneto-one" processing for specific categories. This method simplified the training process and obtained better recognition results with a small amount of training data. In recognition experiments on 2699 images of fruits and vegetables (15 categories), the recognition accuracy rate reached $85 \%$. Koslowski et al. (2013) used MPEG-7 color and texture descriptors from image patches to classify fruits and evaluated classification effect multi-layer perceptron network and support vector machine. In the experiments, the misjudgment rates of 320 fruit images (8 categories) were less than $10 \%$. Biswas and Hossain (2013) extracted and analyzed multiple recognition clues (such as colour, shape, size, texture and weight) of the captured images to classify and recognize the vegetables. The results show that it has high classification accuracy and is suitable for vegetable classification in different occasions.

As shown in Fig. 6, (Zhang et al., 2014) proposed a hybrid classifier that combines the chaos artificial bee colony algorithm and the feedforward neural network, which extracted color, texture and shape features to recognize 18 categories of fruit images, with a recognition accuracy of $89.1 \%$. Considering the impact of different lighting on the images of vegetables and fruits, Dubey and Jalal (2015) extracted different state-of-art color and texture features and combined them to achieve more efficient and discriminative feature description and used multi-class support vector machine to classify different fruit images and the recognition accuracy rate is up to $90.6 \%$.

In order to solve the problem that traditional recognition methods are greatly affected by light, shadows and other factors, Xiaolin et al. (2014) replaced the SVM classifier with a compressed perceptron and merged the extracted feature information to achieve classification. The results show that the highest recognition rate of fruit and vegetable images with 13 categories is 96.2\%. Wang et al. (2016) used ANN-BP model for estimating vegetation chlorophyll content based on red edge parameter, which could greatly improve the accuracy (Yao et al., 2009). used machine vision and neural networks to sort the quality and shape of potatoes.

\section{State Evaluation}

Watcharabutsarakham and Methasate (2019) proposed a system based on the SVM trained on color and Gray Level co-occurrence Matrix (GLCM) image features, which can automatically detect the position of Planthoppers (RPHs) in the collected images and classify the life stage of each hopper and its classification accuracy achieved $87 \%$. By using decision tree 
algorithm, classify analytic data to get images of RPHs, Tsai et al. (2017) proposed a Region Of Interest (ROI) method to detect RPHs, with an $80 \%$ classification accuracy. Yue et al. (2016) also proposed a digital image processing method, based on a nonlinear dispersive phase operation and a multi-feature fusion mean shift method, to segment rice RPHs on curtain in paddy fields. Ma et al. (2013) used OSTU algorithm to segment the embryo images, extracted six characteristic parameters from the images and used the k-means clustering algorithm to identify maize varieties. In the experiment, the variety identification rates are than $94.12 \%$.

Lavania and Matey (2015) combined double thresholding based on the 3D-Otsu's method and the Principal Component Analysis (PCA) method to detect and classify weed in crop rows and it was suited for the real time applications. As shown in Fig. 7, for the detection and characterization of Nitrogen (N) deficiencies in corn fields, Zermas et al. (2015) used small-scale Unmanned Aerial Vehicles (UAVs) and Computer Vision algorithms to analyze the visual (RGB) spectrum and supervised learning methods to characterize crop leaves. The test results show that the accuracy of identifying Ndeficient leaves was $84.2 \%$. Based on the hyperspectral technology, Wu et al. (2019) established Recurrent Neural Network (GRNN) and Probabilistic Neural Network (PNN) to identify the phenological period of cantaloupe fruit.

\section{Navigation of Agricultural Machinery}

Based on monocular vision, Wang et al. (2013) used threshold segmentation and morphological algorithms to extract crops and fitted the running route to realize automatic tractor navigation. Hiremath et al. (2014) proposed a visual navigation algorithm based on the Particle Filter and the experimental results show that the algorithm was robust. As shown in Fig. 8, (Radcliffe et al., 2018) used orchard images captured by a multispectral camera, focusing on tree canopy and sky of an orchard row, to realize the navigation of unmanned ground vehicles.

Qingkuan et al. (2016) used K-means algorithm to extract green crops from the soil background, morphological filtering algorithm to eliminate the weed interference information and Particle Swarm Optimization (PSO) to quickly and accurately detect the navigation line. Zhai et al. (2016) proposed a method for detecting crop rows based on binocular vision with Census transformation and used the Principle Component Analysis (PCA) method to fit the centerline of the corresponding crop row. Zhiyan et al. (2017) proposed a path-planning method based on machine vision for tea crop walking, which combined $2 \mathrm{G}-\mathrm{R}-\mathrm{B}$ processing and the method of least squares.

\section{Deep Learning in Agricultural Engineering}

In recent years, the application and research of Convolutional Neural Networks (CNNs) to agricultural engineering has rapidly increased. The convolutional neural network was inspired by the biological visual cortex. In 1962, Canadian neurologists conducted experiments on the visual cortex of animals and found that some neuronal cells in the brain would make respond when the edges in a specific direction appeared. This mode in which specific neuronal cells will find specific features to complete visual tasks has become the basis of $\mathrm{CNN}$. In recent years, the application of convolutional neural networks and deep learning in agricultural engineering has increased.

\section{Plant Diseases and Insect Pests}

In the recognition of plant diseases and insect pests, the deep learning model effectively improves its accuracy and accuracy. Siyu et al. (2020) used machine vision and adaptive convolutional neural network to detect the quality of peanut kernels and realized the recognition of peanut defects such as mildew, broken and shriveled. Ferentinos (2018) developed convolutional neural network models to perform plant disease detection and diagnosis, with the best performance reaching a $99.53 \%$ success rate.

Min et al. (2019) applied the different combinations of normalizing image size, truncating region of interest and Faster R-CNN Inception v2 (or SSD MobileNet v1) to intelligent diagnosis of rice pests and diseases. DeChant et al. (2017) used deep learning models to effectively identify Northern Leaf Blight (NLB) in maize with $96.7 \%$ accuracy. Chen et al. (2019) constructed an intelligent identification system in process of feature automatic learning, feature fusion, classification and position regression based on deep learning method, the recognition rates of 16 kinds of common pests in light traps under natural condition ranged from 66 to $90 \%$ and the recognition rates of 38 pest symptoms in the field ranged from 50 to $90 \%$. Liu et al. (2018) proposed a deep learning model based on the AlexNet model to detect apple leaf diseases.

Fuentes et al. (2017) presented deep-learning-based approach to detect diseases and pests (Fig. 9) in tomato plants using images captured in-place by camera devices with various resolutions, with three detectors: Faster R-CNN, R-FCN, SSD and additionally propose a method for local and global class annotation and data augmentation to increase the accuracy and reduce the number of false positives during training. Zhang et al. 
(2019a) proposed a deep convolutional neural network method by combining the spatial pyramid pooling with the improved YOLOv3 model and the average accuracy was $88.07 \%$. Barbedo (2018) discussed the main factors that affect the design and effectiveness of deep neural nets applied to plant pathology and built a database of 50,000 images for research.

\section{Agricultural Production}

At present, deep learning is developing rapidly in the field of agriculture and there are a variety of deep learning models and methods that have been applied to various agricultural and food production challenges (Kamilaris and Prenafeta-Boldú, 2018). Cai et al. (2018) exploited a machine learning model based on Deep Neural Network (DNN) and high-performance computing for intelligent and scalable computation of classification processes, which is useful for in-season classification of field-level crop types. Lin et al. (2019) proposed a rice planthopper image classification algorithm based on transfer learning and Mask R-CNN, with a average classification accuracy of $92.3 \%$.

By using a convolutional neural network, (Dyrmann et al., 2016) proposed a method that is capable of recognizing plant species in color images and the network could achieve a classification accuracy of $86.2 \%$ for these 22 species. Zhong et al. (2019) developed a deep learning based classification framework for remotely sensed time series. Kussul et al. (2017) used deep learning models, including a traditional fully connected Multilayer
Perceptron (MLP) and the most commonly used approach in RS community random forest and compare them with $\mathrm{CNNs}$, to classify ground crops from multitemporal multisource satellite imagery.

As show in Fig. 10, Sa et al. (2016) proposed a multimodal Faster R-CNN model for fruits recognition, which is applied to an autonomous agricultural robotic platform. Ye et al. (2019) established a model of stunning state classification based on Faster R-CNN and the recognition accuracy was $96.51 \%$, which can be used to enhance automated slaughtering processes in the poultry industry.

As shown in Fig. 11, Yu et al. (2019) proposed a deep learning method based on the Mask R-CNN. which can automatically detect ripe and unripe strawberries. As show in Fig. 12, Tian et al. (2019) proposed an improved YOLO-V3 model for detecting apples during different growth stages in orchards with fluctuating illumination, complex backgrounds, overlapping apples and branches and leaves. The average detection time of the model is $0.304 \mathrm{~s}$ per frame at $3000 \times 3000$ resolution. Dias et al. (2018) used CNNs to estimate bloom intensity of apple trees, with recall and precision rates higher than $90 \%$. Zhonghong et al. (2019) proposed a new method for fresh grade recognition of spinach based on hyperspectral and deep learning. Maryam et al. proposed an improved Inception-ResNet model for yield estimation, as shown in Fig. 13, with a $91 \%$ average test accuracy on real images and $93 \%$ on synthetic images (Rahnemoonfar and Sheppard, 2017).
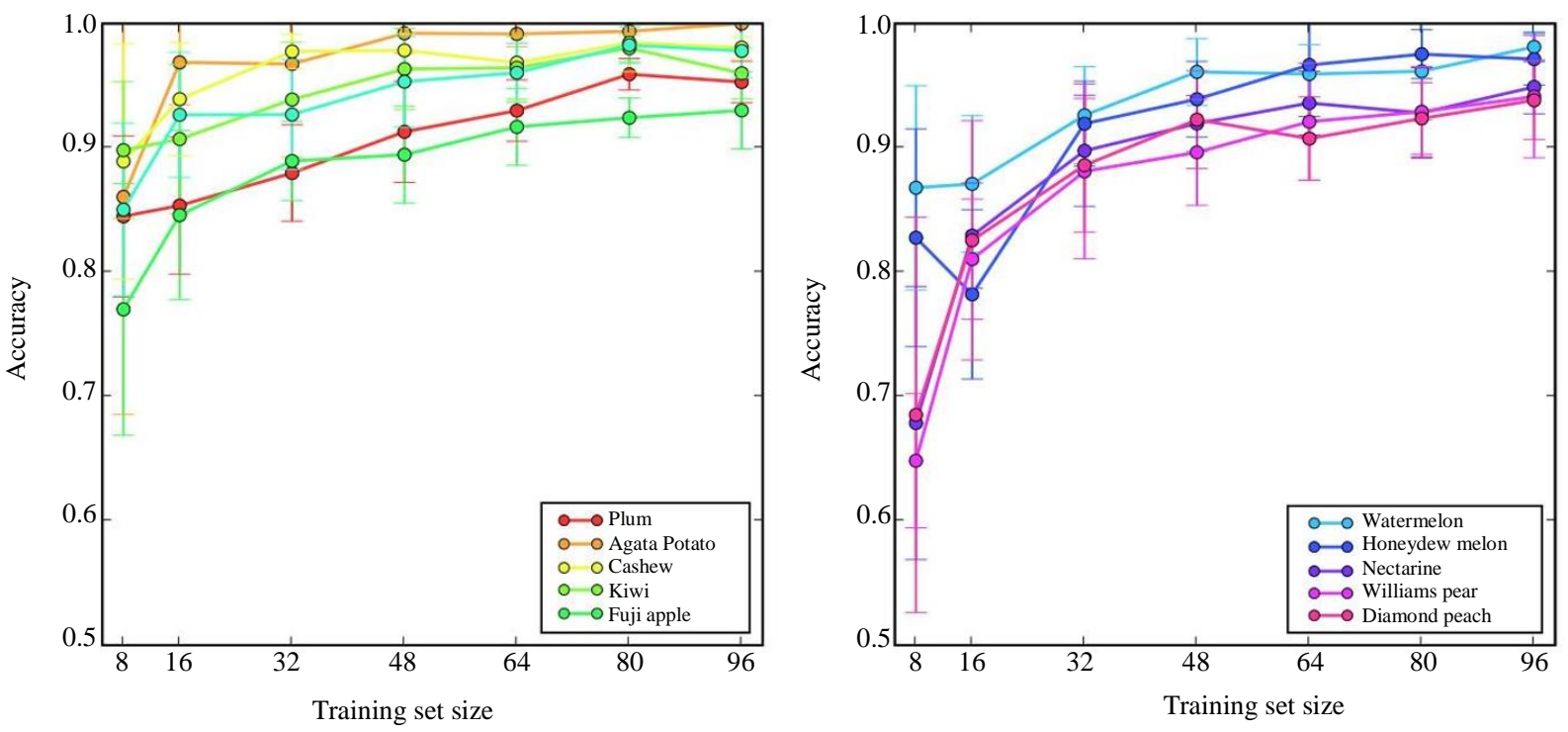

Fig. 5: Average accuracy per class 


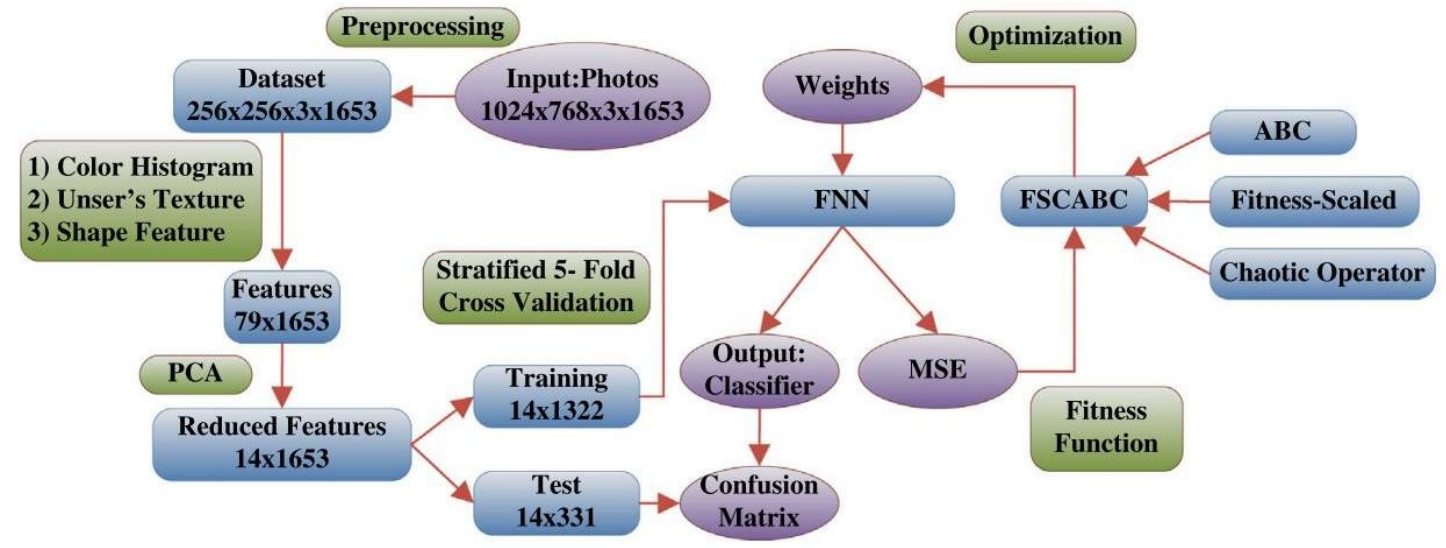

Fig. 6: The flowchart of the proposed fruit recognition system

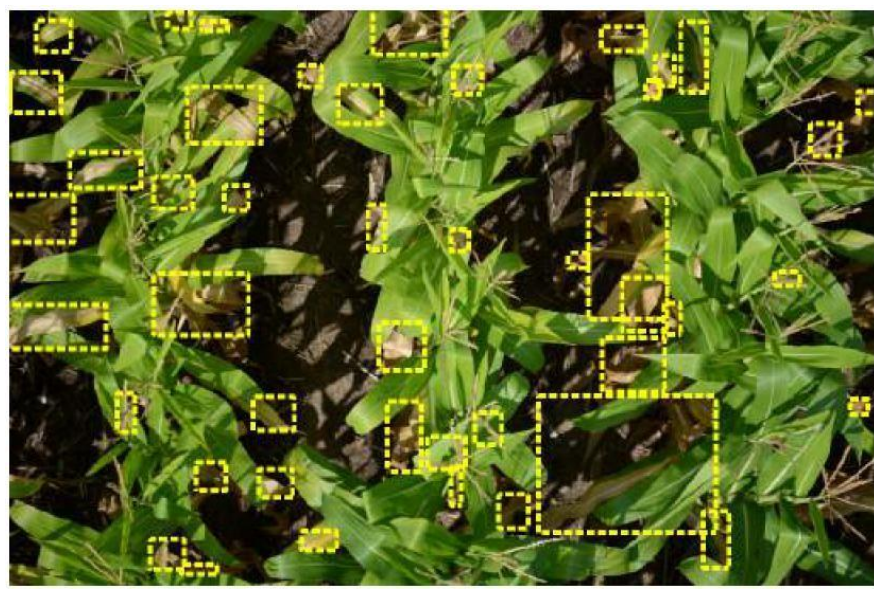

Fig. 7: The output of the recommendation scheme on a heavily N-deficient image
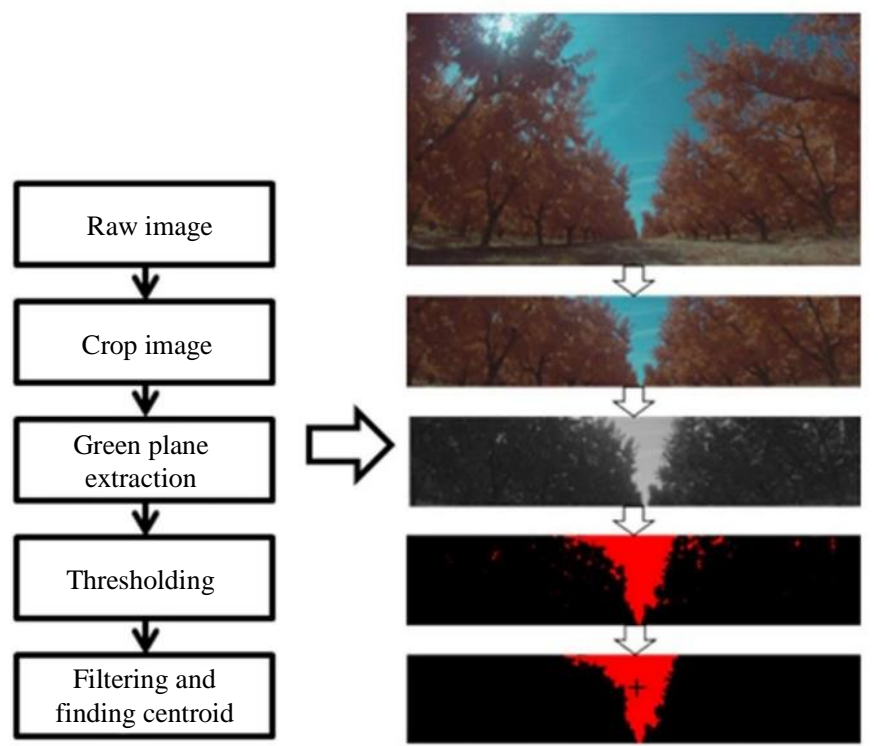

Fig. 8: Image processing algorithm for autonomous navigation 


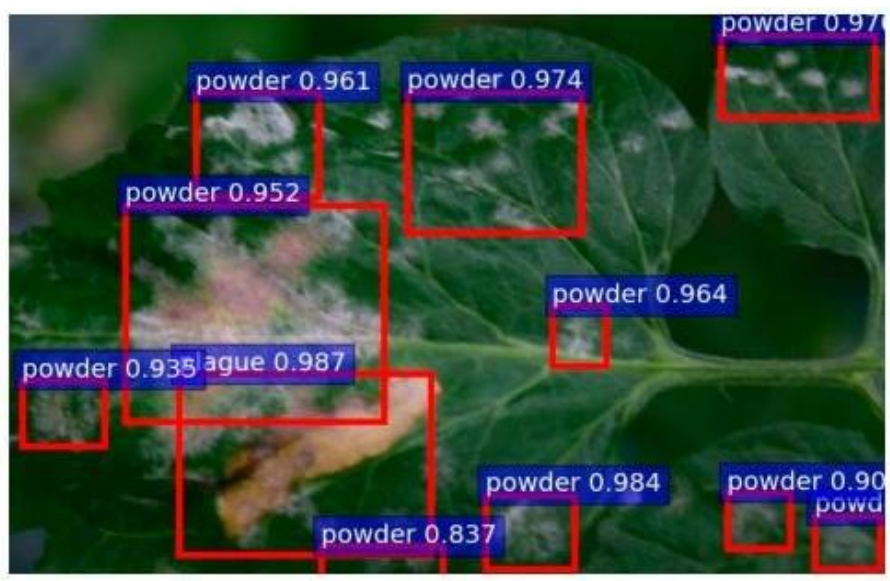

Fig. 9: Detection results of diseases and pests

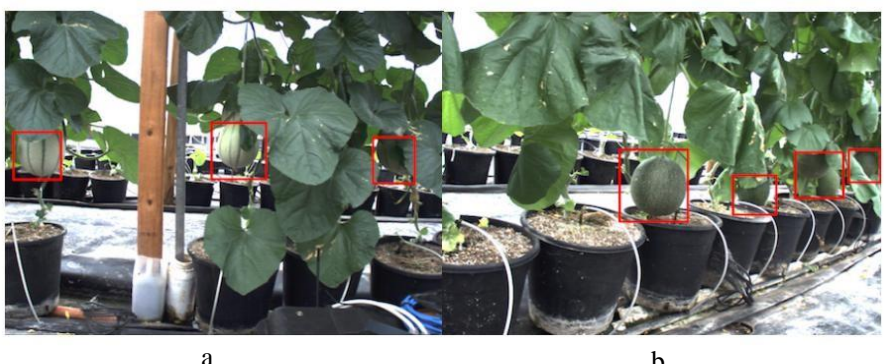

b

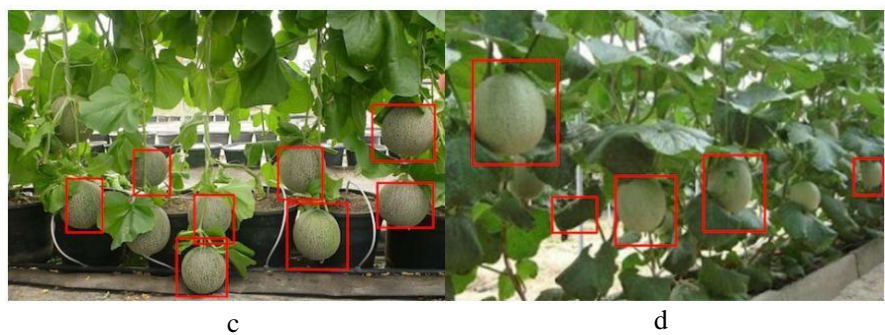

Fig. 10: Fruits recognition based on Faster R-CNN

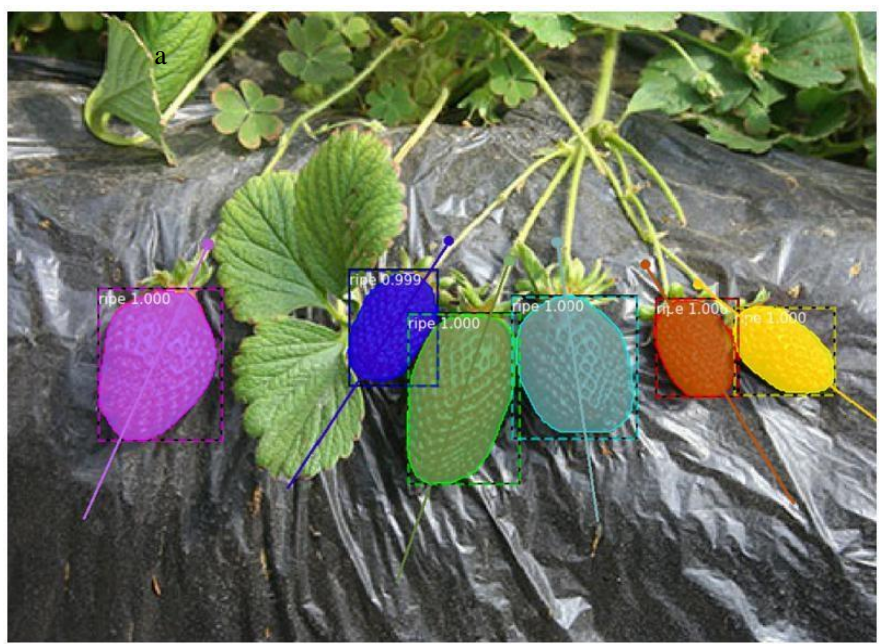

Fig. 11: Strawberry recognition based on Mask R-CNN 

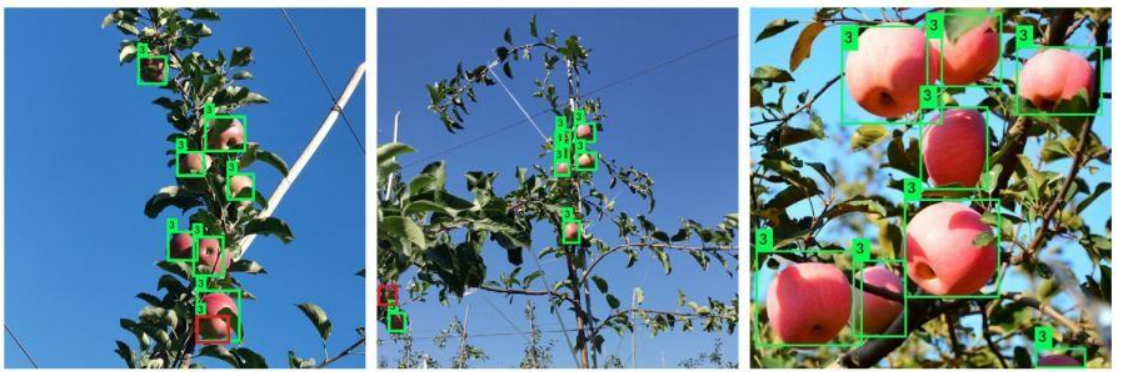

Fig. 12: Apple recognition based on an improved YOLO-V3
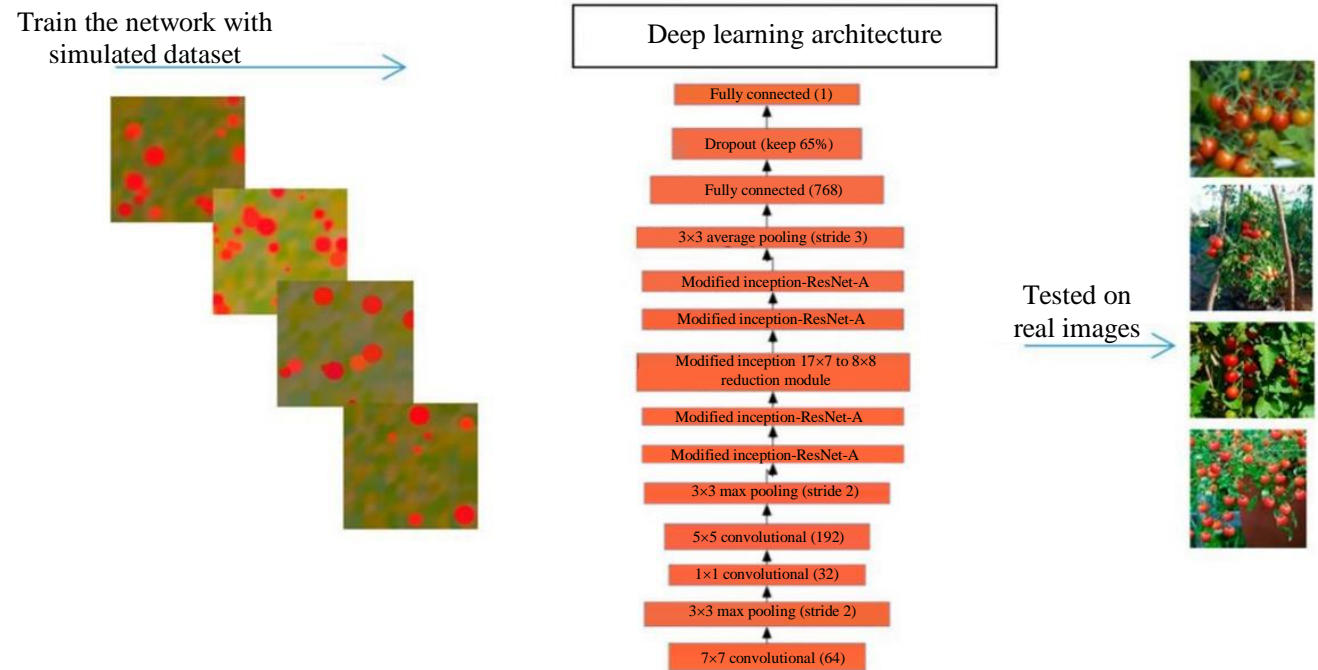

Fig. 13: The framework of the improved Inception-ResNet model foryield estimation

Table 1: Bionic application of animal morphological and movement

\begin{tabular}{lll}
\hline Aspects & Objects & Results and applications \\
\hline Bionic desorption theory & Earthworms and soil animals & Theoretical basis for drag reduction and energy saving \\
Farming machineries & Shells, dung beetles, pangolins, zokors, etc. & Bionic plow-moldboard, bionic press roller and bionic subsoiler \\
Crop cutters & Beetles, beetles, moles, locusts, etc. & Bionic cutters \\
Movement structure & Buffalos, cheetahs, ostrichs, etc. & Bionic walking wheels and robots \\
\hline
\end{tabular}

\section{Discussion and Future Prospect}

In recent years, the research of bionic technology has become more and more extensive and achieved great success in agricultural engineering applications. Bionic technology has gradually developed from the primary imitation, such as shape, structure and movement of animals, to intelligent methods and algorithms, such as machine vision, neural networks and deep learning. Agriculture, as an important industry related to human survival, is the cornerstone of maintaining social stability. Through the development and application of bionic technology, agricultural engineering has been improved: Higher operating efficiency, energy consumption saving, intelligent identification and management. Bionic technology and deep learning have accelerated the innovation of agricultural technology, reduced the number of field staff, increased the output of agricultural products and promoted the automation and intelligence of agricultural engineering.

\section{Bionic Design of Agricultural Machineries}

As an earlier bionic technology used in agriculture, the imitation of soil animal morphology and movement has promoted the development and application of new farm tools and agricultural machinery. The bionic research on morphology and kinematics mainly focuses on farming machinery and cutting cutters. The soil drag reduction of bionic agricultural machineries is conducive to reducing the energy consumption and wear of agricultural machinery during the cultivation process and the bionic design of cutting and crushing tools can reduce energy consumption and improve work efficiency Table 1 . The bionic research and application of animal morphological and movement in agricultural engineering focuses on the following aspects: 
(1) Research on the bionic desorption theory: By exploring characteristics and rules of soil animal desorption phenomenon, the bionic desorption theory provides a theoretical basis for the bionic design of agricultural machinery. The proposal of "biological non-smooth drag reduction theory" and "multiple bionic coupling theory" promoted applications of bionics in agricultural engineering

(2) Design and research of farming machineries: Bionic technology has played an important role in the design of agricultural machinery, effectively solving problems of farming machineries, such as large resistance, serious wear and high energy consumption. By imitating the characteristics of shells, dung beetles, pangolins, zokors and other animal, some bionic plowmoldboard, bionic press roller and bionic subsoiler have characteristics of anti-adhesion and anti-resistance, which promotes the development of agricultural farming machineries

(3) Research on cutting cutters: bionic technology has greatly promoted thedesign and application of crop harvesters. The cutting and crushing cutters imitated the mouthparts of longhorn beetles, the upper jaws of scarabs, the paws of moles and the chewing teeth of locusts are superior to traditional cutting cutters in cutting force and cutting power consumption and improve cutting efficiency

(4) Research on bionic walking wheels and robots: Due to the wheel structure of traditional agricultural machineries, slipping and sinking problems often occur in soft and muddy farmland. Bionic walking wheels and robots have better performances of motion, shock absorption and smoothness. With the development of robot technology, biomimetic agricultural robots are increasingly popularized

\section{Agricultural Recognition and Analysis}

The research and application of machine vision and neural networks in the field of agricultural engineering have broadened the agricultural engineering and it has become an important part of intelligent agriculture. As advanced bionic technology, machine vision and neural network are different from the early bionics (imitating animal characteristics and behaviors), but carried out from visual capture, analysis and processing. In the field of agricultural identification, machine vision and neural networks have played a great role and are widely used in crop classification, quality sorting, variety identification, phenological phase identification, weed identification and route navigation. Machine vision focuses on visual capture, image processing and data analysis and neural networks imitate brain neurons for training and learning. The combination of machine vision and neural networks realizes advanced visual identification in the agricultural field. Machine vision and neural networks are widely used in agricultural engineering, including:
(1) Crop classification and recognition: Machine vision and neural networks can replace manual work to achieve efficient and automatic classification and recognition. A variety of classifiers can be constructed through image processing and neural networks. Classifier construction methods mainly include: Based on feature parameters (such as color, texture, shape, size and edge), support vector machines, multi-layer perception networks and artificial neural networks. The classifiers based on machine vision and neural network accelerate the intelligent recognition in agricultural engineering

(2) Size and quality sorting: Automatic sorting of size and quality effectively reduces time consumption and labor costs. Machine vision combined with neural network, OSTO algorithm, K-means clustering algorithm, feature dimension reduction and other algorithms can sort crops (such as potatoes, corn) and further realize the sorting of weeds and crops, identify weeds and automate weeding

(3) Phenological phase recognition: Machine vision combined with chromatograph, spectroscopy and regression neural network can identify crop phenological phases, evaluating growth status and yield, which is very important in agricultural engineering applications

(4) Path extraction and navigation: The path of crops can be extracted through machine vision to realize autonomous navigation of agricultural machinery. Vision acquisition methods include monocular cameras, binocular cameras and depth cameras. Machine vision combined with neural networks, Kmeans clustering algorithm and other algorithms can extract path information such as crop ranks and walking paths between crops

\section{Intelligent Agricultural Engineering}

In recent years, the rapid development of deep learning has promoted the intelligentization of agricultural engineering. As a more advanced bionic technology, deep learning is more complex than machine vision and artificial neural networks, but it can solve classification problems more efficiently and accurately. Compared with the basic bionic technology, deep learning requires more calculations and faster processing speed. With the development of integrated circuit and chip technology, the research and application of deep learning are increasing. Deep learning consists of multi-layer networks for feature extraction, pooling and output. Common deep learning models include CNN, R-CNN, Faster R-CNN, R-FCN, SSD, Mask R-CNN, YOLO, etc. The research and application of deep learning in agricultural engineering focus on the following aspects: 
(1) Classification: Deep learning has played an important role in the recognition and classification and of crops. By building huge databases for learning and training, it can quickly and accurately classify crop varieties, independent of target characteristics and lighting environment

(2) Recognition of pests and diseases: After learning and training, the deep learning model has become an important means of identifying pests and diseases, speeding up the diagnosis speed and accuracy

(3) Evaluation of growth status: Deep learning can identify and evaluate the maturity, flowering, freshness and yield of crops to achieve intelligent management

(4) Intelligent robots: Intelligent robots based on deep learning are the most popular research direction, leading the development of automation and intelligence in agricultural engineering. The combination of deep learning, robotics and other disciplines enables agricultural machineries more intelligent, such as perception and autonomous navigation and it will accelerate the development of intelligent agricultural robots

Deep learning is an important method of artificial intelligence and its application promoted the development of intelligent agriculture. Compared with machine vision and neural networks, deep learning models have higher accuracy and efficiency. Bionic technology has developed from simple imitation design to intelligent processing and its application in agricultural engineering has changed from agricultural machineries and cutters to intelligent recognition and autonomous agricultural robots.

With the development of bionic technology and deep learning, the improvement of agricultural engineering will not only be the optimization of agricultural machinery and crop identification, but also intelligent management and unmanned monitoring of agriculture. Although deep learning has promoted agricultural intelligence, the characteristics of high cost and complicated operation are still problems to be solved. Bionic technology and deep learning will bring new challenges and opportunities to agricultural practitioners and will continue to promote the development of agricultural technology. The research of bionic technology still needs continuous exploration and suitable and convenient intelligent agricultural robots will become the hotspot of future research.

\section{Conclusion}

This paper discusses the agricultural engineering application of bionic technology and deep learning technology in detail. The bionic technology has played an important role in promoting the agricultural development. Traditional bionics technology is widely used in agriculture, such as reducing soil resistance of agricultural machinery and improvement of cutting cutters. These applications indicate that bionic technology has incomparable advantages in agricultural engineering. Machine vision and neural networks are widely used in agricultural engineering to solve the problems of crop classification, sorting, phenological period recognition and navigation. In recent years, deep learning has been deeply explored and applied in agricultural engineering, especially in the aspects of crop classification, disease and pest diagnosis, growth status evaluation and autonomous robots, showing high efficiency and intelligence.

At present, bionic technology and deep learning are still important methods and driving forces of agricultural development and more in-depth research is needed. At the same time, deep learning still has many problems, such as time-consuming, high cost and high complexity. With more in-depth research, bionic technology and deep learning will be improved and optimized continually, leading to intelligent and sustainable agriculture.

\section{Acknowledgement}

This work was supported by the Key Technology Project of prevention and control of major accidents in production safety [grant numbers: jiangsu-0002-2017AQ].

This work was supported by the Scientific and Technological Projects of quality and technical supervision Bureau of Jiangsu province [grant numbers: KJ175933].

\section{Author's Contributions}

Chunlei Tu and Jie Li: Contributed significantly to analysis and manuscript preparation.

Jinxia Li and Cheng Shen: Participated to collect the materials.

Xingsong Wang: Contributed to the conception of the study.

\section{Ethics}

The authors declare their responsibility for any ethical issues that may arise after the publication of this manuscript.

\section{Conflict of Interest}

The authors declare that they have no competing interests. The corresponding author affirms that all of the authors have read and approved the manuscript.

\section{References}

Ananthanarayanan, A., Azadi, M., \& Kim, S. (2012). Towards a bio-inspired leg design for high-speed running. Bioinspiration \& Biomimetics, 7(4), 046005. https://iopscience.iop.org/article/10.1088/17483182/7/4/046005/meta 
Barbedo, J. G. (2018). Factors influencing the use of deep learning for plant disease recognition. Biosystems Engineering, $\quad 172, \quad 84-91$. https://www.sciencedirect.com/science/article/abs/pi i/S1537511018303027

Berling, J., \& Rechberger, M. (2007). Knives as sharp as rat's teeth. Research News, 1 . www.fraunhofer.de/fhg/EN/press/pi/2005/01/Medie ndienst012005Thema3.jsp

Biswas, H., \& Hossain, F. (2013). Automatic vegetable recognition system. International Journal of Engineering Science Invention, 2(4), 377-41.

Cai, Y., Guan, K., Peng, J., Wang, S., Seifert, C., Wardlow, B., \& Li, Z. (2018). A high-performance and in-season classification system of field-level crop types using time-series Landsat data and a machine learning approach. Remote sensing of environment, 210, 35-47.

https://www.sciencedirect.com/science/article/abs/pi i/S0034425718300610

Chen, D. H., Tong, J., Li, C. H., Zhang, S. J., \& Chen, B. C. (2003). A review of man and animal gait and walking robot. Journal of Jilin University: Engineering and Technology Edition, 33(4), 121-125. http://en.cnki.com.cn/Article_en/CJFDTOTALJLGY200304027.htm

Chen, T., Zeng, J., Xie, C., Wang, R., Liu, W., Zhang, J., ... \& Dong, W. (2019). Intelligent identification system of disease and insect pests based on deep learning. China Plant Protection, 39(04), 26-34. http://en.cnki.com.cn/Article_en/CJFDTotalZBJS201904006.htm

DeChant, C., Wiesner-Hanks, T., Chen, S., Stewart, E. L., Yosinski, J., Gore, M. A., ... \& Lipson, H. (2017). Automated identification of northern leaf blightinfected maize plants from field imagery using deep learning. Phytopathology, 107(11), 1426-1432. https://apsjournals.apsnet.org/doi/full/10.1094/PHY TO-11-16-0417-R

Dias, P. A., Tabb, A., \& Medeiros, H. (2018). Apple flower detection using deep convolutional networks. Computers in Industry, 99, 17-28. https://www.sciencedirect.com/science/article/abs/pi i/S016636151730502X

Dickinson, M. H. (1999). Bionics: Biological insight into mechanical design. Proceedings of the National Academy of Sciences, 96(25), 14208-14209. https://www.pnas.org/content/96/25/14208.short

Dubey, S. R., \& Jalal, A. S. (2015). Fruit and vegetable recognition by fusing colour and texture features of the image using machine learning. International Journal of Applied Pattern Recognition, 2(2), 160-181. https://www.inderscienceonline.com/doi/abs/10.150 4/IJAPR.2015.069538
Dyrmann, M., Karstoft, H., \& Midtiby, H. S. (2016). Plant species classification using deep convolutional neural network. Biosystems Engineering, 151, 72-80. https://www.sciencedirect.com/science/article/abs/pi i/S1537511016301465

Faria, F. A., dos Santos, J. A., Rocha, A., \& Torres, R. D. S. (2012, August). Automatic classifier fusion for produce recognition. In 2012 25th SIBGRAPI Conference on Graphics, Patterns and Images (pp. 252-259). IEEE.

https://ieeexplore.ieee.org/abstract/document/6382764

Ferentinos, K. P. (2018). Deep learning models for plant disease detection and diagnosis. Computers and Electronics in Agriculture, 145, 311-318. https://www.sciencedirect.com/science/article/abs/pi i/S0168169917311742

Fuentes, A., Yoon, S., Kim, S. C., \& Park, D. S. (2017). A robust deep-learning-based detector for real-time tomato plant diseases and pests recognition. Sensors, 17(9), 2022. https://www.mdpi.com/14248220/17/9/2022

Hiremath, S., Van Evert, F. K., ter Braak, C., Stein, A., \& van der Heijden, G. (2014). Image-based particle filtering for navigation in a semi-structured agricultural environment. Biosystems Engineering, 121, 85-95. https://www.sciencedirect.com/science/article/abs/pi i/S1537511014000312

Honglei, J., Mingzhuo, G., \& Chunjiang, G. (2018). Design of dynamic bionic stubble cutting device and optimization test of parameters. Transactions of the Chinese Society for Agricultural Machinery, 49(10), 103-114.

http://en.cnki.com.cn/Article_en/CJFDTotalNYJX201810012.htm

Ji, W., Jia, H., \& Tong, J. (2012). Experiment on working performance of bionic blade for soil-rototilling and stubble-breaking. Transactions of the Chinese Society of Agricultural Engineering, 28(12), 24-30. https://www.ingentaconnect.com/content/tcsae/tcsae /2012/00000028/00000012/art00005

Jia, H., Ji, W., Han, W., Tan, H., Liu, Z., \& Ma, C. (2009). Optimization experiment of structure parameters of rototilling and stubble breaking universal blade. Nongye Jixie Xuebao= Transactions of the Chinese Society for Agricultural Machinery, 40(7), 45-50. https://www.cabdirect.org/cabdirect/abstract/2009 3231027

Jia, H., Li, C., Zhang, Z., \& Wang, G. (2013). Design of bionic saw blade for corn stalk cutting. Journal of Bionic Engineering, 10(4), 497-505. https://link.springer.com/article/10.1016/S16726529(13)60242-5 
Jia, H., Wang, Q., Huang, D., Zhu, L., Li, M., \& Zhao, J. (2019). Design of bionic mole forelimb intelligent row cleaners. International Journal of Agricultural and Biological Engineering, 12(3), 27-35. http://www.ijabe.org/index.php/ijabe/article/view/4408

Kamilaris, A., \& Prenafeta-Boldú, F. X. (2018). Deep learning in agriculture: A survey. Computers and Electronics in Agriculture, 147, 70-90. https://www.sciencedirect.com/science/article/abs/pi i/S0168169917308803

Kang, I. S., \& Sams, A. R. (1999). Bleedout efficiency, carcass damage and rigor mortis development following electrical stunning or carbon dioxide stunning on a shackle line. Poultry Science, 78(1), 139-143. https:/www.sciencedirect.com/science/article/pii/S0 03257911941273X

Koslowski, M. A., Santos, F. G., Borba, G. B., \& Gamba, H. R. (2013). Fruits Classification Using MPEG-7 Descriptors from Image Patches. In IX Workshop de Visão Computacional (WVC 2013). http://iris.sel.eesc.usp.br/wvc/Anais_WVC2013/Oral/3/ 4.pdf

Kussul, N., Lavreniuk, M., Skakun, S., \& Shelestov, A. (2017). Deep learning classification of land cover and crop types using remote sensing data. IEEE Geoscience and Remote Sensing Letters, 14(5), 778-782. https://ieeexplore.ieee.org/abstract/document/7891032

Lavania, S., \& Matey, P. S. (2015, February). Novel method for weed classification in maize field using Otsu and PCA implementation. In 2015 IEEE International Conference on Computational Intelligence \& Communication Technology (pp. 534-537). IEEE. https://ieeexplore.ieee.org/abstract/document/7078760

LeCun, Y., Bengio, Y., \& Hinton, G. (2015). Deep Learning. Nature, 521(7553), 436-444. https://www.nature.com/articles/nature14539

Li, A., Qian, C., Liancheng, W., Luquan, R., \& Zhizhong, W. (1998). Bionics Design for Scaly Nonsmooth Surfaces. Journal of Jilin University of Technology. Vol. 28(2), 12-16 http://en.cnki.com.cn/Article_en/CJFDTOTALJLGY802.002.htm

Li, J., Yan, Y., Chirende, B., Wu, X., Wang, Z., \& Zou, M. (2017). Bionic design for reducing adhesive resistance of the ridger inspired by a boar's head. Applied Bionics and Biomechanics, 2017. https://www.hindawi.com/journals/abb/2017/8315 972/

Lin, X., Zhu, S., Zhang, J., \& Liu, D. (2019). Rice planthopper image classification method based on transfer learning and mask R-CNN. Transactions of the Chinese Society for Agricultural Machinery, 50(07), 201-207.

http://en.cnki.com.cn/Article_en/CJFDTotal-

NYJX201907021.htm
Liu, B., Zhang, Y., He, D., \& Li, Y. (2018). Identification of apple leaf diseases based on deep convolutional neural networks. Symmetry, 10(1), 11. https://www.mdpi.com/2073-8994/10/1/11

Liu, S., Weng, S., Liao, Y., \& Zhu, D. (2014). Structural bionic design for digging shovel of cassava harvester considering soil mechanics. Applied Bionics and Biomechanics, $\quad 11(1, \quad 2), \quad 1-11$. https://content.iospress.com/articles/applied-bionicsand-biomechanics/abb089

Luquan, R., \& Yunhong, L. (2016). The Introduction of Bionics. Beijing: Science Press (in Chinese).

Ma, D., Cheng, H., \& Zhang, W. (2013, December). Maize embryo image acquisition and variety identification based on OTSU and K-means clustering algorithm. In 2013 International Conference on Information Science and Cloud Computing Companion (pp. 835-840). IEEE. https://ieeexplore.ieee.org/abstract/document/6973696

Min, J., Yiming, S., Jingyao, Z., Yuan, R., \& Wei, D. (2019). Research on Rice Diseases and Pests Diagnosis Based on Deep Learning. Journal of Luoyang Institute of Science and Technology (Natural Science Edition), 29(04), 78-83 (in Chinese with English abstract) http://en.cnki.com.cn/Article_en/CJFDTotalLYGY201904014.htm

Patel, K. K., Kar, A., Jha, S. N., \& Khan, M. A. (2012). Machine vision system: a tool for quality inspection of food and agricultural products. Journal of Food Science and Technology, 49(2), 123-141. https://link.springer.com/article/10.1007/s13197011-0321-4

Qingkuan, M., Man, Z., \& Genghuang, Y. (2016). Guidance line recognition of agricultural machinery based on particle swarm optimization under natural illumination. Transactions of the Chinese Society for Agricultural Machinery, 47(6), 11-20.

Radcliffe, J., Cox, J., \& Bulanon, D. M. (2018). Machine vision for orchard navigation. Computers in Industry, 98, 165-171.

https://www.sciencedirect.com/science/article/abs/pi i/S0166361517305389

Rahnemoonfar, M., \& Sheppard, C. (2017). Deep count: fruit counting based on deep simulated learning. Sensors, 17(4), 905. https://www.mdpi.com/1424$8220 / 17 / 4 / 905$

Rocha, A., Hauagge, D. C., Wainer, J., \& Goldenstein, S. (2008, October). Automatic produce classification from images using color, texture and appearance cues. In 2008 XXI Brazilian Symposium on Computer Graphics and Image Processing (pp. 3-10). IEEE. https://ieeexplore.ieee.org/abstract/document/4654137/ 
Rocha, A., Hauagge, D. C., Wainer, J., \& Goldenstein, S. (2010). Automatic fruit and vegetable classification from images. Computers and Electronics in Agriculture, $\quad$ 70(1), 96-104. https:/www.sciencedirect.com/science/article/abs/pi i/S016816990900180X

Sa, I., Ge, Z., Dayoub, F., Upcroft, B., Perez, T., \& McCool, C. (2016). Deepfruits: A fruit detection system using deep neural networks. Sensors, 16(8), 1222. https://www.mdpi.com/1424-8220/16/8/1222

Schmidhuber, J. (2015). Deep learning in neural networks: An overview. Neural Networks, 61, 85-117. https://www.sciencedirect.com/science/article/abs/pi i/S0893608014002135

Seng, W. C., \& Mirisaee, S. H. (2009, August). A new method for fruits recognition system. In 2009 International conference on electrical engineering and informatics (Vol. 1, pp. 130-134). IEEE. https://ieeexplore.ieee.org/abstract/document/5254804

Seok, S., Wang, A., Chuah, M. Y., Otten, D., Lang, J., \& Kim, S. (2013, May). Design principles for highly efficient quadrupeds and implementation on the MIT Cheetah robot. In 2013 IEEE International Conference on Robotics and Automation (pp. 3307-3312). IEEE. https://ieeexplore.ieee.org/abstract/document/6631038

Siyu, Z., Qiuju, Z., \& Ke, L. (2020). Detection of peanut kernel quality based on machine vision and adaptive convolution neural network. Transactions of the Chinese Society of Agricultural Engineering (Transactions of the CSAE), 36(4), 269-277. http://www.cnki.com.cn/Article/CJFDTotalNYGU202004032.htm

Tian, K., Li, X., Shen, C., Zhang, B., Huang, J., Wang, J., \& Zhou, Y. (2017). Design and test of cutting blade of cannabis harvester based on longicorn bionic principle. Transactions of the Chinese Society of Agricultural Engineering, 33(5), 56-61. https://www.ingentaconnect.com/content/tcsae/tcsae /2017/00000033/00000005/art00008

Tian, Y., Yang, G., Wang, Z., Wang, H., Li, E., \& Liang, Z. (2019). Apple detection during different growth stages in orchards using the improved YOLO-V3 model. Computers and Electronics in Agriculture, 157, 417-426. https://www.sciencedirect.com/science/article/abs/pi i/S016816991831528X

Tong, J., Ji, W., Jia, H., Chen, D., \& Yang, X. (2015). Design and tests of biomimetic blades for soil-rototilling and stubble-breaking. Journal of Bionic Engineering, 12(3), 495-503.

https://www.sciencedirect.com/science/article/abs/pii/S 1672652914601402

Tsai, T. H., Lee, T. Y., \& Chen, P. H. (2017, May). The ROI of rice planthopper by image processing. In 2017 International Conference on Applied System Innovation (ICASI) (pp. 126-129). IEEE. https://ieeexplore.ieee.org/abstract/document/7988363
Wang, G., Jia, H., Zhao, J., Li, C., Wang, Y., \& Guo, H. (2014). Design of corn high-stubble cutter and experiments of stubble retaining effects. Transactions of the Chinese Society of Agricultural Engineering, 30(23), 43-49. https://www.ingentaconnect.com/content/tcsae/tcsae /2014/00000030/00000023/art00006

Wang, H. C., Ming, T. U., Nian, L., Rong, Z., Zhongming, W., \& Jin, T. (2018). Burrowing Activities of Zokor and Quantitative Analysis of Geometric Features of Its Claws. Journal of Anhui Agricultural Ences, 46(9), 165-170 (in Chinese with English abstract) http://en.cnki.com.cn/Article_en/CJFDTotalAHNY201809065.htm

Wang, H., Xiong, J., Li, Z., Deng, J., \& Zou, X. (2016). Potato grading method of weight and shape based on imaging characteristics parameters in machine vision system. Transactions of the Chinese Society of Agricultural Engineering, 32(8), 272-277. https://www.ingentaconnect.com/content/tcsae/tcsae /2016/00000032/00000008/art00038

Wang, Y., Yang, F., Wang, T., Liu, Q., \& Xu, X. (2013). Research on visual navigation and remote monitoring technology of agricultural robot. International Journal on Smart Sensing \& Intelligent Systems, 6(2). https://www.exeley.com/exeley/journals/in_jour_sm art_sensing_and_intelligent_systems/6/2/pdf/10.213 07_ijssis-2017-550.pdf

Watcharabutsarakham, S., \& Methasate, I. (2019). Mobiledevice Based Image Processing for Rice Brown Planthopper Classification and Outbreak Monitoring. Applied Engineering in Agriculture, 35(1), 15-21. https://elibrary.asabe.org/abstract.asp?aid=50018

Wei, W., Fei, D., Fengwei, Z., Wuyun, Z., Shilin, Z., Ruij, S. (2018). Design of bionic harvest cutting table of flax in dry area. Journal of Chinese Agricultural Mechanization, 39(08), 44-48 (in Chinese with English abstract) http://en.cnki.com.cn/Article_en/CJFDTotalGLJH201808010.htm

Wu, H., Mingzhou, L., Hang, Z., Dongmei, P., Zexin, L., \& Ling, Z. (2019). Dentification model of fruit phenology of cantaloupe based on hyperspectral technique. Jiangsu Agricultural Sciences, 47(22), 258-264 (in Chinese). http://www.cnki.com.cn/Article/CJFDTotalJSNY201922061.htm

Xiaolin, H., Yueju, X., Shuqin, T., Hongsheng, L., \& Jinhui, H. (2014). Rgb-d images classification based on compressed sensing theory. Computer Applications and Software, 31(3), 195-198 (in Chinese with English abstract) http://en.cnki.com.cn/Article_en/CJFDTOTALJYRJ201403052.htm 
Xu, Z., Zhao, J., Wu, Z. H., \& Shi, H. (2009). Analysis of drag reduction on dimple concave non-smooth surface. Aviation Precision Manufacturing Technology, 45(1), 33-38. https://en.cnki.com.cn/Article_en/CJFDTotalHJZJ200901013.htm

Xue, J., Zhang, L., \& Grift, T. E. (2012). Variable fieldof-view machine vision based row guidance of an agricultural robot. Computers and Electronics in Agriculture, $\quad 84, \quad 85-91$. https://www.sciencedirect.com/science/article/abs/pi i/S016816991200049X

Xue, W. (2017). Design and Research of Bionic Drag Reduction Double Disc Opener. Master thesis, Jilin University (in Chinese with English abstract). http://cdmd.cnki.com.cn/Article/CDMD-101831017160836.htm

Yanhu, H., Dianlei, H., Guoyu, L., Gang, L., \& Rui, Z. (2018). Bionic Design and Performance Analysis of Adaptive Low Vibration Walking Wheel. Transactions of the Chinese Society for Agricultural Machinery, 49(3), 418-426 (in Chinese with English abstract) http://en.cnki.com.cn/Article_en/CJFDTotalNYJX201803053.htm

Yao, F., Zhang, Z., Yang, R., Sun, J., \& Cui, S. (2009). Hyperspectral models for estimating vegetation chlorophyll content based on red edge parameter. Transactions of the Chinese Society of Agricultural Engineering, 25(1), 123-129. https:/www.ingentaconnect.com/content/tcsae/tcsae /2009/00000025/A00201s2/art00024

Ye, C., Rui, K., Chao, Q., Chao, L., Yang, Z., Kunjie, C. (2019). Stunning State Recognition Method of Broiler Chickens Based on Faster Region Convolutional Neural Network. Transactions of The Chinese Society of Agricultural Machinery, 50(12), 255-259 (in Chinese with English abstract). http://en.cnki.com.cn/Article_en/CJFDTotalNYJX201912029.htm

Yongxiang, L. (2004). Significance and Progress of Bionics. Journal of Bionic Engineering, 1(1), 1-3. https://link.springer.com/article/10.1007\%2FBF033 99448

Yu, Y., Zhang, K., Yang, L., \& Zhang, D. (2019). Fruit detection for strawberry harvesting robot in nonstructural environment based on Mask-RCNN. Computers and Electronics in Agriculture, 163, 104846. https://www.sciencedirect.com/science/article/abs/pi i/S0168169919301103

Yue, H., Man, H., Wang, K., \& Cao, L. (2016, August). Rice planthopper segmentation based on multifeature fusion. In 2016 IEEE International Conference on Signal and Image Processing (ICSIP) (pp; 16-21). IEEE. https://ieeexplore.ieee.org/abstract/document/7888215
Zermas, D., Teng, D., Stanitsas, P., Bazakos, M., Kaiser, D., Morellas, V., ... \& Papanikolopoulos, N. (2015, September). Automation solutions for the evaluation of plant health in corn fields. In 2015 IEEE/RSJ International Conference on Intelligent Robots and Systems (IROS) (pp. 6521-6527). IEEE. https://ieeexplore.ieee.org/abstract/document/7354309

Zhai, Z., Zhu, Z., Du, Y., Zhang, S., \& Mao, E. (2016). Method for detecting crop rows based on binocular vision with Census transformation. Transactions of the Chinese Society of Agricultural Engineering, 32(11), 205-213.

https://www.ingentaconnect.com/content/tcsae/tcsae /2016/00000032/00000011/art00029

Zhang, B., Zhang, M., \& Chen, Y. (2019a). Crop pest identification based on spatial pyramid pooling and deep convolution neural network. Transactions of the Chinese Society of Agricultural Engineering (Transactions of the CSAE), 35(19), 209-215.

Zhang, D., Guobiao, Z., Jin, T., Zhihong, Z. (2019b). Development of device for bionic sub-soil liquid fertilizer. Transactions of the Chinese Society of Agricultural Engineering, 35(19), 29-36 (in Chinese with English abstract). http://en.cnki.com.cn/Article_en/CJFDTotalNYGU201919004.htm

Zhang, J. (2014). Bionic theory and technology of drag reduction and wear resistance of subsoil shovel. Doctoral thesis, Jilin University (in Chinese).

Zhang, N., Wang, M., \& Wang, N. (2002). Precision agriculture-a worldwide overview. Computers and Electronics in agriculture, 36(2-3), 113-132. https://www.sciencedirect.Com/science/article/abs/p ii/S0168169902000960

Zhang, Y., Wang, S., Ji, G., \& Phillips, P. (2014). Fruit classification using computer vision and feedforward neural network. Journal of Food Engineering, 143, 167-177. https://www.sciencedirect.com/science/article/abs/pi i/S026087741400291X

Zhiyan, M., Yousheng, T., Guangyou, Y., (2017). Research of Tea Garden Path Planning Based on Machine Vision. Journal of Agricultural Mechanization Research, 39(1), 202-206 (in Chinese with English abstract).

Zhong, L., Hu, L., \& Zhou, H. (2019). Deep learning based multi-temporal crop classification. Remote sensing of environment, 221, 430-443. https://www.sciencedirect.com/science/article/abs/pi i/S0034425718305418

Zhonghong, X., Huanliang, X., \& Qiugui, H. (2019). Spinach freshness detection based on hyperspectral image and deep learning method. Transactions of the Chinese Society of Agricultural Engineering (Transactions of the CSAE), 35(13), 277-284. 